\title{
Synchronization transition in scale-free networks: Clusters of synchrony
}

\author{
Deok-Sun Lee \\ Theoretische Physik, Universität des Saarlandes, 66041 Saarbrücken, Germany
}

(Dated: October 30, 2018)

\begin{abstract}
We study the synchronization transition in scale-free networks that display power-law asymptotic behaviors in their degree distributions. The critical coupling strength and the order-parameter critical exponent derived by the mean field approach depend on the degree exponent $\lambda$, which implies a close connection between structural organization and the emergence of dynamical order in complex systems. We also derive the finite-size scaling behavior of the order parameter finding that the giant cluster of synchronized nodes is formed in different ways between scale-free networks with $2<\lambda<3$ and those with $\lambda>3$.
\end{abstract}

PACS numbers: 89.75.-k, 05.45.Xt, 05.70.Fh

\section{INTRODUCTION}

Much attention has recently been paid to integrative approaches to complex systems in diverse fields. Graphtheoretic analyses on social, biological, and informational networks consisting of relevant elements (nodes) and interactions (edges) have found common features such as short average distance between nodes, high clustering, and scalefree (SF) topology, which means that the degree distribution is of a power-law form [1, 2]. These structural characteristics have been found to be evidence of competitive evolution processes [3] as well as ground for the robustness of complex systems against external attacks or internal errors [4]. However, the relation of the structural organization to dynamical complexity remains to be explored. Recent works on epidemic spreading [5], avalanche dynamics [6, 7], and reaction-diffusion processes [8, 9] in complex networks identified the significance of network topology in dynamic behavior. Here we focus on collective synchronization phenomena that appear in various physical and biological systems including Josephson junction arrays, cardiac pacemaker cells, flashing fireflies, and brains [10] as well as are required in controlling artificial systems [11]. While each element of these systems can be described simply by a limit-cycle oscillator, the coupling topologies are not always regular. For instance, the neuronal network with synaptic coupling in human brains turns out to have SF topology [12, 13], whose synchrony is essential in the recognition of spatio-temporal patterns [14]. Also in parallel simulations where tasks are distributed among many processing elements, weak random couplings among them can prevent the spread of virtual times from diverging [11]. Our interest is in how the emergence of dynamical order is intertwined with structural complexity.

The linear stability analysis of the synchronized state in complex networks of oscillators has shown that the synchronizability, quantified by the ratio of the largest and the second smallest eigenvalues of the Laplacian matrix, would be suppressed by a few "center" nodes being overloaded by the traffic of communication [15, 16, 17]. However, the effects of topological features on the phase transition from the desynchronized state to the synchronized state demand further investigation. In small-world (SW) networks [1], the synchronization transition is observed at a finite critical coupling strength and associated critical exponents are shown to be equal to those of the globally-coupled case [18, 19, 20] meaning both belong to the same universality class [21]. In this paper we report on the synchronization transition in SF networks of limit-cycle oscillators. The degree distribution of the SF networks takes a power-law form $p_{d}(k) \sim k^{-\lambda}$ for $k \gg 1$. We find by the mean-field approach that the critical coupling strength is much smaller than those of completely random graphs [22] or SW networks [1] that have Poisson degree distributions, and the order-parameter critical exponent varies continuously with the degree exponent $\lambda$ as long as $2<\lambda<5$, illustrating the relation between the structural organization and the emergence of dynamical order. We also show how the mean-field approach enables to compute the order parameter in the critical regime for finite system size and derive the finite-size scaling behavior of the order parameter finding different nature of the synchronization transition between SF networks with $2<\lambda<3$ and $\lambda>3$. Our numerical data confirm the analytic results.

The paper is organized as follows. The model for the coupled limit-cycle oscillators on a network is introduced and the order parameter is defined in the following section. In Sec. IIII we use the mean-field approach to set up self-consistent equations and find the critical point and order-parameter critical exponent. The finite-size scaling behavior is derived in Sec. IV We summarize and discuss our work in Sec. V

\section{MODEL AND ORDER PARAMETER}

We consider $N$ coupled limit-cycle oscillators whose phases $\left\{\phi_{i}(t) \mid i=1,2, \ldots, N\right\}$ evolve according to

$$
\frac{d \phi_{i}}{d t}=\omega_{i}-\frac{J}{\langle k\rangle} \sum_{j=1}^{N} e_{i j} \sin \left(\phi_{i}-\phi_{j}\right),
$$

where $J$ is the coupling strength, $e_{i j}=1(0)$ if oscillators $i$ and $j$ are connected (disconnected), and $\langle k\rangle$ denotes the average degree $\sum_{i} k_{i} / N$ with $k_{i}=\sum_{j} e_{i j}$. The natural frequencies $\omega_{i}$ 's are distributed following the Gaussian probability distribution $g\left(\omega_{i}\right)=(2 \pi)^{-1 / 2} e^{-\omega_{i}^{2} / 2}$. A system of globally-coupled oscillators evolving by Eq. (1), i.e., the case of $e_{i j}=1$ for all $i \neq j$ and $\langle k\rangle=N-1$, defines the Kuramoto model [18] and has been used as an exactly solvable model of collective synchro- 


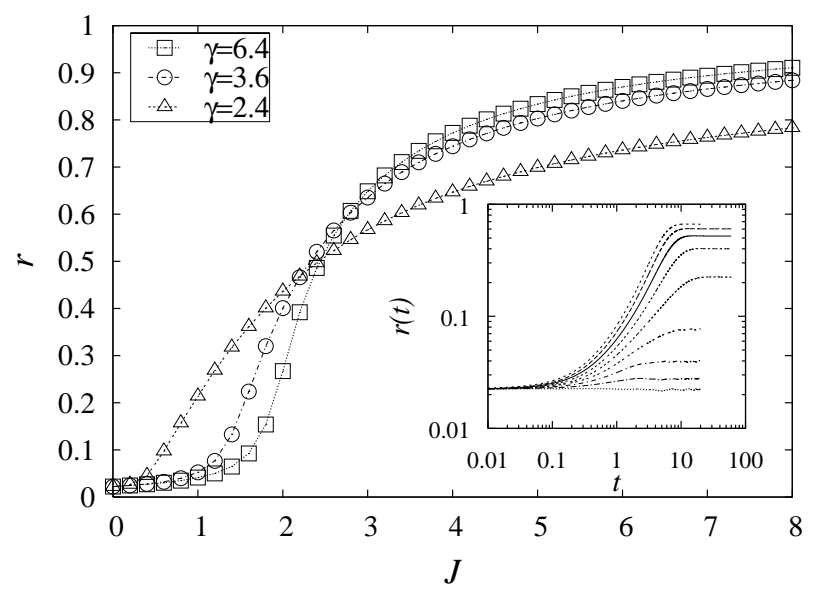

FIG. 1: Order parameter $r=\lim _{t \rightarrow \infty} r(t)$ versus the coupling strength $J$ for $N=1600,\langle k\rangle=4$, and $\lambda=6.4,3.6$, and 2.4. Inset: Time evolution of $r(t)$ for $\lambda=3.6$ with $J=0.0,0.4,0.8,1.2,1.6,2.0,2.4,2.8$ and 3.2 from bottom to top.

nization [10]. Here we are interested in sparse SF coupling networks with $\langle k\rangle=O(1)$ and $\lambda>2$.

Synchronization may be quantified by the amplitude order parameter $r(t)$ defined by

$$
r(t) e^{i \theta(t)}=\frac{1}{N} \sum_{j=1}^{N} e^{i \phi_{j}(t)}
$$

The average phase $\theta(t)$ satisfies $d \theta(t) / d t=\bar{\omega}$ with $\bar{\omega}=$ $(1 / N) \sum_{i=1}^{N} \omega_{i}$ for a given frequency realization $\left\{\omega_{i}\right\}$. To compute $r(t)$, we performed a numerical integration of Eq. (1) on SF networks that are generated using the static model introduced in Ref. [23]. $r(t)$ is averaged over several hundreds of different frequency and network realizations, $\left\{\omega_{i}\right\}$ and $\left\{e_{i j}\right\}$. As shown in the inset of Fig. 1] $r(t)$ saturates to a value $r$ in the long-time limit. We plot $r$ for $N=1600$ as a function of $J$ in Fig. 11 evaluated by $r=(2 / T) \sum_{t=T / 2+1}^{T} r(t)$, where we set $T$ so as to guarantee that the saturation of $r$ has already begun at time $T / 2$. The numerical results imply that the transition from the desynchronized state $(r=0)$ to the synchronized state ( $r$ non-zero) in the thermodynamic limit $N \rightarrow \infty$ exhibits a dependence on the coupling topology characterized by the degree exponent. The critical phenomena related to the synchronization transition in SF networks can be understood by the mean-field approach.

\section{SELF-CONSISTENT EQUATIONS AND CRITICAL EXPONENT}

In this section, we obtain the mean-field solution to Eq. (1) and find the critical point and order-parameter critical exponent. Assuming the same magnitude of effective field between each pair of coupled oscillators, one obtains the onebody differential equation from Eq. (1) as $d \phi_{i} / d t=\omega_{i}-$
$(J /\langle k\rangle) k_{i} \bar{r}(t) \sin \phi_{i}$, where the effective field $\bar{r}(t)$ satisfies

$$
\bar{r}(t) e^{i \bar{\theta}(t)}=\frac{\sum_{i=1}^{N} \sum_{j=1}^{N} e_{i j} \exp \left(i \phi_{j}\right)}{\sum_{i=1}^{N} \sum_{j=1}^{N} e_{i j}},
$$

and the relation $\bar{\theta}(t) \rightarrow 0$ in the thermodynamic limit is used. $\bar{r}(t)$ will be computed in a self-consistent way.

In the stationary state, the oscillators are either locked or drifting [18, 19]. For given $\bar{r}=\lim _{t \rightarrow \infty} \bar{r}(t)$, locked oscillators have their frequencies in the region $\left|\omega_{i}\right| \leq J k_{i} \bar{r} /\langle k\rangle$ and thus their phases are locked at $\sin ^{-1}\left(\omega_{i}\langle k\rangle /\left(J k_{i} \bar{r}\right)\right)$ while the frequencies of drifting oscillators are in the region $\left|\omega_{i}\right|>$ $J k_{i} \bar{r} /\langle k\rangle$ and thus $d \phi_{i} / d t \neq 0$. Drifting oscillators do not contribute to the order parameter due to the symmetry of the frequency distribution $g(\omega)=g(-\omega)$. Thus the order parameter $r$ is computed only in terms of locked oscillators as

$r=\sum_{k=1}^{\infty} p_{d}(k) \int_{-\frac{J k \bar{r}}{\langle k\rangle}}^{\frac{J k \bar{r}}{\langle k\rangle}} d \omega g(\omega) \int_{0}^{2 \pi} d \phi e^{i \phi} \delta\left[\phi-\sin ^{-1}\left(\frac{\omega\langle k\rangle}{J k \bar{r}}\right)\right]$.

The effective field $\bar{r}$ is obtained by solving the following selfconsistent equation derived from its definition,

$\bar{r}=\sum_{k=1}^{\infty} \frac{k p_{d}(k)}{\langle k\rangle} \int_{-\frac{J k \bar{r}}{\langle k\rangle}}^{\frac{J k \bar{r}}{\langle k\rangle}} d \omega g(\omega) \int_{0}^{2 \pi} d \phi e^{i \phi} \delta\left[\phi-\sin ^{-1}\left(\frac{\omega\langle k\rangle}{J k \bar{r}}\right)\right]$.

To solve Eqs. (4) and (5), we expand them in powers of $\bar{r}$ as

$$
r=\sum_{n=1}^{\infty} a_{2 n-1}\left(\frac{J}{\langle k\rangle} \bar{r}\right)^{2 n-1} \text { and } \bar{r}=\sum_{n=1}^{\infty} \bar{a}_{2 n-1}\left(\frac{J}{\langle k\rangle} \bar{r}\right)^{2 n-1}
$$

which are valid for finite $N$. Here the coefficients are given by $a_{2 n-1}=\left\langle k^{2 n-1}\right\rangle u_{2 n-1}$ and $\bar{a}_{2 n-1}=\left(\left\langle k^{2 n}\right\rangle /\langle k\rangle\right) u_{2 n-1}$, where $\left\langle k^{m}\right\rangle=\sum_{k} k^{m} p_{d}(k)$, and

$$
u_{2 n-1}=\frac{(-1)^{n-1} 2^{-n-1 / 2}(n-3 / 2) !}{n !(n-1) !},
$$

from the relation $g(\omega)=(2 \pi)^{-1 / 2} \sum_{n=0}^{\infty}\left(-\omega^{2} / 2\right)^{n} / n !$ and $\int_{-\pi / 2}^{\pi / 2} d \phi \cos ^{2} \phi \sin ^{2 n-2} \phi=\pi^{1 / 2}(n-3 / 2) ! /(2 n !)$. Eq. (6) is valid also in the thermodynamic limit unless $a_{2 n-1}$ or $\bar{a}_{2 n-1}$ diverge. In case of SF networks with $p_{d}(k) \sim k^{-\lambda}$, however, $\left\langle k^{m}\right\rangle$ diverges as $N^{m /(\lambda-1)-1} /(m-\lambda+1)$ for $m>$ $\lambda-1$ [24] and thus $a_{2 n-1}(n>\lambda / 2)$ and $\bar{a}_{2 n-1} \quad(n>$ $(\lambda-1) / 2)$ diverge: $a_{2 n-1} \simeq c_{2 n-1} N^{(2 n-1) /(\lambda-1)-1} /(2 n-\lambda)$ for $n>\lambda / 2$ and $\bar{a}_{2 n-1} \simeq \bar{c}_{2 n-1} N^{(2 n) /(\lambda-1)-1} /(2 n-\lambda+1)$ for $n>(\lambda-1) / 2$ with $c_{2 n-1}$ and $\bar{c}_{2 n-1}$ constants. In terms of a scaling variable $q \equiv N^{1 /(\lambda-1)} J \bar{r} /\langle k\rangle$, the terms with such diverging coefficients in Eq. (6) are arranged as $(J \bar{r} /\langle k\rangle)^{\lambda-1} \sum_{n>\lambda / 2} c_{2 n-1} q^{2 n-\lambda} /(2 n-\lambda)=(J \bar{r} /\langle k\rangle)^{\lambda-1} f_{\lambda}(q)$ for $r$ and $(J \bar{r} /\langle k\rangle)^{\lambda-2} \sum_{n>(\lambda-1) / 2} \bar{c}_{2 n-1} q^{2 n-\lambda+1} /(2 n-\lambda+$ $1)=(J \bar{r} /\langle k\rangle)^{\lambda-2} \bar{f}_{\lambda}(q)$ for $\bar{r}$. The alternating sign and fast decay of $c_{2 n-1}$ and $\bar{c}_{2 n-1}$ from the behavior of $u_{2 n-1}$ in Eq. (7) make $f_{\lambda}(q)$ and $\bar{f}_{\lambda}(q)$ finite in the limit $q \rightarrow \infty$ that corresponds to $N \rightarrow \infty$ and $\bar{r}$ finite. Consequently, the following 
equations should be considered for SF networks in the thermodynamic limit,

$$
\begin{aligned}
& r=\sum_{n=1}^{\infty} a_{2 n-1}^{\prime}\left(\frac{J}{\langle k\rangle} \bar{r}\right)^{2 n-1}+a_{\lambda-1}^{\prime}\left(\frac{J}{\langle k\rangle} \bar{r}\right)^{\lambda-1}+\cdots, \\
& \bar{r}=\sum_{n=1}^{\infty} \bar{a}_{2 n-1}^{\prime}\left(\frac{J}{\langle k\rangle} \bar{r}\right)^{2 n-1}+\bar{a}_{\lambda-2}^{\prime}\left(\frac{J}{\langle k\rangle} \bar{r}\right)^{\lambda-2}+\cdots,
\end{aligned}
$$

where all the coefficients are finite and in particular, $a_{2 n-1}^{\prime}=$ $a_{2 n-1}$ for $n \leq\lfloor\lambda / 2\rfloor$ and $\bar{a}_{2 n-1}^{\prime}=\bar{a}_{2 n-1}$ for $n \leq\lfloor(\lambda-1) / 2\rfloor$ with $\lfloor x\rfloor$ denoting here the greatest integer that is less than $x$. Notice that $a_{\lambda-1}^{\prime}=f_{\lambda}(\infty)$ and $\bar{a}_{\lambda-2}^{\prime}=\bar{f}_{\lambda}(\infty)$ [25].

The existence of the synchronization transition for $\lambda>3$ is identified by the emergence of non-zero values of $r$ and $\bar{r}$ only when $\bar{a}_{1}^{\prime} J /\langle k\rangle>1$ or $J>J_{c}$ with

$$
J_{\mathrm{c}}=2 \sqrt{\frac{2}{\pi}} \frac{\langle k\rangle^{2}}{\left\langle k^{2}\right\rangle} .
$$

Inspecting Eq. (8) for $0<\Delta \equiv J / J_{c}-1 \ll 1$, one finds that $r \simeq\left(\langle k\rangle^{2} /\left\langle k^{2}\right\rangle\right) \bar{r}$ and that $\Delta \bar{r} \simeq\left|\bar{a}_{3}^{\prime}\right|\left(J_{c} \bar{r} /\langle k\rangle\right)^{3}$ for $\lambda>5$ and $\Delta \bar{r} \simeq\left|\bar{a}_{\lambda-2}^{\prime}\right|\left(J_{c} \bar{r} /\langle k\rangle\right)^{\lambda-2}$ for $3<\lambda<5$. Thus it holds for small positive $\Delta$ and $\lambda>3$ that

$$
r \sim \Delta^{\beta}
$$

with the order-parameter critical exponent $\beta$ given by

$$
\beta= \begin{cases}\frac{1}{2} \quad \text { for } \lambda>5 \\ \frac{1}{\lambda-3} \text { for } 3<\lambda<5 .\end{cases}
$$

Notice that in the globally-coupled case where $\left\langle k^{2}\right\rangle=\langle k\rangle^{2}=$ $(N-1)^{2}$, the critical point $J_{c}$ is $2 \sqrt{2 / \pi} \simeq 1.60$ and the critical exponent $\beta$ is $1 / 2$ [18, 19]. In Ref. [26], the exponent $\beta$ has been shown numerically to be close to $1 / 2$ in the synchronization transition on the Barabási-Albert network [3] that has $\lambda=3$.

When $2<\lambda<3,\left\langle k^{2}\right\rangle$ is $O\left(N^{(3-\lambda) /(\lambda-1)}\right)$ [24] and thus the critical point $J_{c}$ is of order $N^{-(3-\lambda) /(\lambda-1)}$, which is zero in the thermodynamic limit. It means that $\bar{r}$ and $r$ are always nonzero values for non-zero values of $J$. Eq. (8) is arranged into $r \simeq 2^{-3 / 2} \pi^{1 / 2} J \bar{r}$ and $\bar{r} \simeq\left|\bar{a}_{\lambda-2}^{\prime}\right|(J \bar{r} /\langle k\rangle)^{\lambda-2}$ for small $J$, which leads to

$$
\bar{r} \sim J^{\frac{\lambda-2}{3-\lambda}} \quad \text { and } \quad r \sim J^{\frac{1}{3-\lambda}} .
$$

The phase diagram and dynamical states of the oscillators in the desynchronized state and the synchronized state are shown in Fig. 2] The only contribution to the order parameter in the desynchronized state is made by temporary synchronization of a few oscillators that could be disconnected. On the contrary, a finite fraction of oscillators have their phases close to the average phase $\theta(t)$ in the synchronized state. This giant cluster of synchronized nodes contains a finite fraction of edges as well, implying that the synchrony originates from the interaction between them through the SF coupling topology. Furthermore, the continuously-varying critical exponent

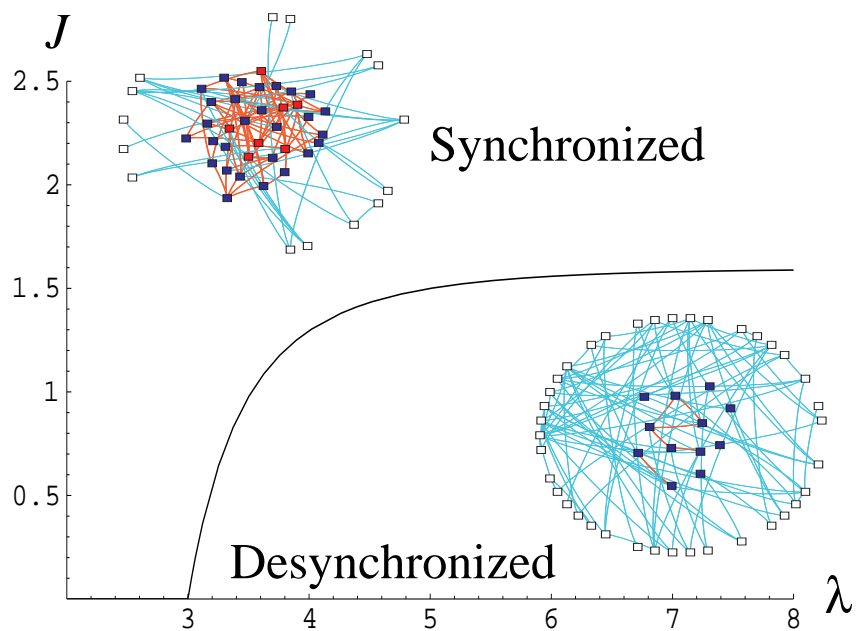

FIG. 2: (Color online) Phase diagram and typical dynamical states of oscillators on SF networks. The phase boundary in Eq. (9) is drawn with $p_{d}(k)=k^{-\lambda} / \zeta(\lambda)$ for $k \geq 1$ and $\zeta(\lambda)$ the Riemann-zeta function. The two network configurations represent dynamical states of coupled oscillators that have evolved with $J=1.0$ (desynchronized) and $J=4.0$ (synchronized) respectively on SF networks with $\lambda=3$.6. In both, synchronized nodes, those having their phases $\phi_{i}$ so close to the average phase $\theta$ that $\left|\phi_{i}-\theta\right|<0.5$, are drawn as filled boxes inside the circle while the others are as empty boxes at the circumference. Edges connecting synchronized nodes are drawn as thick lines.

$\beta$ according to the degree exponent $\lambda$ demonstrates that the variation of the network topology may bring about the change of the universality class of the synchronization transition on complex networks. The influence of the structural organization on the emergence of dynamical order is more significant in case of $2<\lambda<3$ where $J_{c}=0$. A deeper understanding of the nature of the synchronization transition is available when we investigate the finite-size scaling behavior of the order parameter. As we shall see, it illuminates different nature of the synchronization transition between SF networks with $2<\lambda<3$ and $\lambda>3$ as well as allows to confirm our findings, Eqs. (9), (10), (11), and (12), numerically.

\section{DERIVATION OF FINITE-SIZE SCALING BEHAVIOR}

In this section, we describe how to compute the order parameter in the critical regime and combining it with the results in the previous section, present the finite-size scaling behavior of the order parameter.

In finite-size systems, the order parameter $r$ is non-zero even with $J=0$ so that $r_{J=0} \sim N^{-1 / 2}$, as the phases are distributed uniformly in $[0,2 \pi)$. With $J$ increasing from $J=$ 0 , the clusters, connected oscillators with nearly the same phases, form and evolve to find the largest one among them much larger than $r_{J=0} N \sim N^{1 / 2}$, when the order parameter $r$ can be approximated by $r \simeq S / N$ with $S$ the (ensembleaveraged) largest cluster size. The synchronized state is marked by the presence of a giant cluster whose size is $O(N)$ 

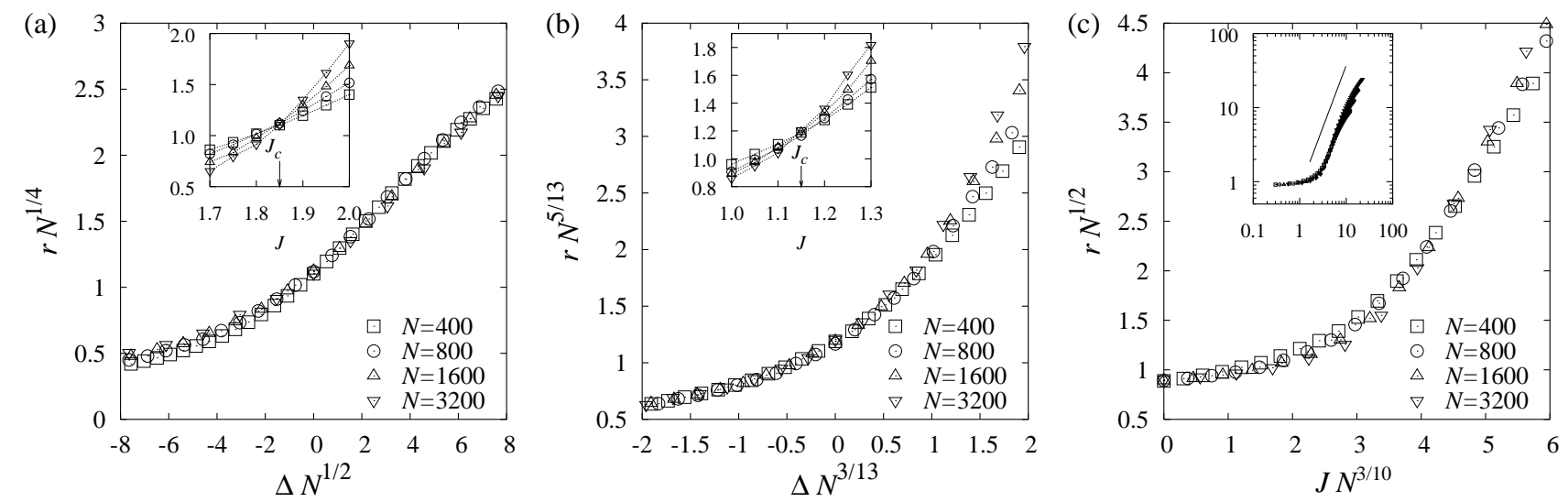

FIG. 3: Data collapse of scaled order parameter for $\langle k\rangle=4$, (a) $\lambda=6.4$, (b) $\lambda=3.6$, and (c) $\lambda=2.4$ according to Eqs. (15) and (16). In (a) and (b), insets show the crossing of scaled order parameters at $J_{c}$, which is found to be (a) 1.85(5) and (b) 1.15(5), larger than 1.22 and 0.84 , respectively, evaluated from Eq. 9. In the inset of (c), asymptotic behavior of the scaling function is explicitly shown. The solid line has slope $1 /(3-\lambda)=5 / 3$.

making $r$ finite.

To understand the jump to $O(1)$ of the order parameter in the critical regime for $\lambda>3$ and in the small $J$ regime for $2<\lambda<3$, we should know the largest cluster size $S_{c}$ in those regimes. $S_{c}$ is of order $N^{\alpha}$ with $1 / 2 \leq \alpha<1$, and we will try to extract the exponent $\alpha$ from the asymptotic behavior of the cluster size distribution, which can be obtained using Eq. (8), but only for $\lambda>3$. For $2<\lambda<3$, we will see that the largest cluster size increases gradually from $O\left(N^{1 / 2}\right)$ to $O(N)$ without any characteristic size between them while $J$ is small.

Let us define the generating functions $\mathcal{P}(z)=\sum_{s} P(s) z^{s}$ and $\overline{\mathcal{P}}(z)=\sum_{s} \bar{P}(s) z^{s}$, where $P(s)$ is the probability that a node belongs to a size-s cluster and $\bar{P}(s)$ the probability that an edge is followed by a size-s cluster. The dependence on the system size $N$ is implicitly included. The generating functions are analytic for finite $N$ and thus one can expand the inverse function $\overline{\mathcal{P}}^{-1}(\omega)$ as $z=\overline{\mathcal{P}}^{-1}(\omega)=1-\sum_{n \geq 1} b_{n}(1-\omega)^{n}$ around $\omega=1$. When $z=z_{N}^{*} \equiv e^{-\frac{1}{S}}$ with $\tilde{S}$ satisfying $S_{2} \ll \tilde{S} \ll S$ and $S_{2}$ being the second largest cluster size, the corresponding value $\omega_{N}^{*}=\overline{\mathcal{P}}\left(z_{N}^{*}\right)$ represents the statistical weight of all clusters but the largest one, that is, $\omega_{N}^{*} \simeq \sum_{s<S} \bar{P}(s)$. Suppose that Eq. (8) is valid in the critical regime. Since $\bar{r}=\lim _{N \rightarrow \infty}\left(1-\omega_{N}^{*}\right)$, one can see that the coefficients $b_{n}$ should take such values as enable the equation $z=1-\sum_{n>1} b_{n}(1-\omega)^{n}$ with $z=\lim _{N \rightarrow \infty} z_{N}^{*}=1$ to be reduced to Eq. (8) with $\bar{r}$ replaced by $1-\omega$. Consequently we have $z=\omega+\sum_{n=1}^{\infty} \bar{a}_{2 n-1}^{\prime}[J(1-$ $\omega) /\langle k\rangle]^{2 n-1}+\bar{a}_{\lambda-2}^{\prime}[J(1-\omega) /\langle k\rangle]^{\lambda-2}+\cdots$ with $\omega=\overline{\mathcal{P}}(z)$.

The last relation informs us that at the critical point where $\bar{a}_{1}^{\prime} J /\langle k\rangle=1,1-\overline{\mathcal{P}}(z)$ has the following leading terms: $(1-$ $z)^{1 / 3}$ for $\lambda>5,(1-z)^{1 /(\lambda-2)}$ for $3<\lambda<5$, and $J^{\lambda-2}(1-$ $z)^{\lambda-2}$ for $2<\lambda<3$, respectively. Eq. (8) also enables us to know that $\mathcal{P}(z)$ is related to $\overline{\mathcal{P}}(z)$ via $1-\mathcal{P}(z) \sim J(1-\overline{\mathcal{P}}(z))$ and thus the leading terms of $1-\mathcal{P}(z)$ are given as $(1-z)^{1 / 3}$ for $\lambda>5,(1-z)^{1 /(\lambda-2)}$ for $3<\lambda<5$, and $J^{\lambda-1}(1-z)^{\lambda-2}$ for $2<\lambda<3$, respectively. Here we showed the $J$ dependence explicitly only for $2<\lambda<3$. These singulari- ties give the asymptotic behaviors of $P(s)$ through the relations $\left.(1 / s !)\left(d^{s} / d z^{s}\right) \mathcal{P}(z)\right|_{z=0}=P(s)$ and $(1 / s !)\left(d^{s} / d z^{s}\right)(1-$ $z)\left.^{\tau-1}\right|_{z=0} \sim s^{-\tau}$ for large $s$. Finally the largest cluster size $S_{c}$ at the critical point can be obtained using the relation $\sum_{s<S_{c}} P(s)=1-S_{c} / N$ [27]. The result is

$$
S_{c} \sim \begin{cases}N^{\frac{3}{4}} & \text { for } \lambda>5, \\ N^{\frac{\lambda-2}{\lambda-1}} & \text { for } 2<\lambda<5 .\end{cases}
$$

For $\lambda>3, S_{c}$ in Eq. (13) is much larger than $r_{J=0} N \sim N^{1 / 2}$ and we can accept it as true. The oscillators are not ready to form the giant cluster until the coupling strength reaches the critical value. There emerge clusters of all sizes up to $S_{c}$ at the critical point and many of them merge into the giant cluster with the coupling strength slightly increased. Combining Eqs. (10) and 13, and introducing another exponent $\mu$ defined by $S_{c} / N \sim N^{-\beta / \mu}$, which is given as

$$
\mu= \begin{cases}2 & \text { for } \lambda>5, \\ \frac{\lambda-1}{\lambda-3} & \text { for } 3<\lambda<5,\end{cases}
$$

we obtain the following finite-size scaling behavior of $r$

$$
r=N^{-\beta / \mu} \Psi\left(\Delta N^{1 / \mu}\right) .
$$

The scaling function $\Psi(x)$ is a constant for $x \ll 1$ while behaves as $x^{\beta}$ for $x \rightarrow \infty$. We remark that $O\left(N^{-1 / 4}\right)$ fluctuation of $r$ at $J=J_{c}$ for $\lambda>5$ has also been found in the Kuramoto model [20] as well as in SW networks [21]. We plot the scaled numerical data for $\lambda=6.4$ and 3.6 in Fig. 3. (a) and (b), respectively, which show good agreement with Eq. (15) except for slight deviations of $J_{c}$ from Eq. (9). Such deviations of the critical point have also been observed in the Monte Carlo simulation of the Ising model on SF networks [28], but are not so serious as to give rise to a finite value of $J_{c}$ even for $2<\lambda<3$. It is known that the critical point can be more precisely predicted by studying the system on the Cayley tree with a given degree distribution [29]. 
When $2<\lambda<3, S_{c}$ in Eq. 13) is much smaller than $r_{J=0} N$. This means that Eq. 8 is not correct in the critical regime because of strong finite-size effects. Instead, the finite-size scaling behavior of the order parameter for $2<\lambda<3$ can be obtained by connecting $r \sim J^{1 /(3-\lambda)}$ in Eq. (12) and $r_{J=0} \sim$ $N^{-1 / 2}$, which leads to

$$
r=N^{-\frac{1}{2}} \Phi\left(J N^{\frac{3-\lambda}{2}}\right)
$$

where $\Phi(x)$ is a constant for $x \ll 1$ and $\Phi(x) \sim x^{1 /(3-\lambda)}$ for $x \rightarrow \infty$. This behavior is confirmed by the numerical data with $\lambda=2.4$ in Fig. 3 (c).

Distinctive finite-size scaling behaviors shown in Eqs. 15 and (16) represent a significant difference in the emergence of dynamical order between SF networks with $\lambda>3$ and $2<\lambda<3$. In SF networks with $2<\lambda<3$, the giant cluster of synchrony is formed if only $J \gg N^{-(3-\lambda) / 2}$. A large number of hub nodes that are connected with one another do not allow separate clusters of various sizes to be developed but force most nodes to belong to the unique giant cluster for any nonzero coupling strength. It is completely different from the way of forming the giant cluster in SF networks with $\lambda>3$. Clusters of various sizes up to $O\left(N^{1-\beta / \mu}\right)$ in the critical regime merge to form the giant cluster as the system escapes from the critical regime. The advantage of many real complex systems having their degree exponents between 2 and 3 in their network structures is thus very obvious. Such highly heterogeneous systems can remain in the dynamically-ordered state only with a very small coupling strength, e.g., $O\left(N^{-(3-\lambda) / 2}\right)$ in the synchronization phenomena, which is required to maintain the stability of the system. On the other hand, a perturbation can propagate easily through the hubs as revealed by the linear stability analysis of the ordered state [15, 16, 17], nec- essary to adapt the system to the changes of the environment.

\section{SUMMARY AND DISCUSSION}

We investigated the synchronization transition of coupled limit-cycle oscillators on complex networks analytically and numerically. We used the mean-field approach to find the critical coupling strength and the critical exponent. Furthermore, we showed how the self-consistent equations derived by the mean-field approach can be used also to compute the critical fluctuation of the order parameter that completes the finite-size scaling analysis. We believe that this method can be generally applied to the study of finite-size effects in complex networks. The synchronization transition turned out to be closely related to the formation of the giant cluster of synchronized oscillators, which crucially depends on the connectivity pattern of a given network such that the critical phenomena are distinguished according to the degree exponent. Our findings imply that complex systems take very heterogeneous connectivity patterns to acquire both stability and flexibility with only a small interaction among the elements. While preparing this manuscript, we have learned of the work by T. Ichinomiya [30], which partly overlaps with ours.

\section{Acknowledgments}

We are indebted to the network group at SNU, where this work was initiated, for helpful discussions. We thank Byungnam Kahng and Heiko Rieger for comments on the manuscript. This work was supported by the Deutsche Forschungsgemeinschaft (DFG).
[1] D.J. Watts and S.H. Strogatz, Nature 393, 440 (1998).

[2] R. Albert and A.-L. Barabási, Rev. Mod. Phys. 74, 47 (2002).

[3] A.-L. Barabási and R. Albert, Science 286, 509 (1999).

[4] R. Albert, H. Jeong, and A.-L. Barabási, Nature 406, 378 (2000).

[5] R. Pastor-Satorras and A. Vespignani, Phys. Rev. Lett. 86, 3200 (2001); Phys. Rev. E 63, 066117 (2001).

[6] D.J. Watts, Proc. Natl. Acad. Sci. U.S.A. 99, 5766 (2002).

[7] K.-I. Goh, D.-S. Lee, B. Kahng, and D. Kim, Phys. Rev. Lett. 91, 148701 (2003); D.-S. Lee, K.-I. Goh, B. Kahng, and D. Kim, J. Korean Phys. Soc. 44, 633 (2004); Physica A 338, 84 (2004).

[8] L.K. Gallos and P. Argyrakis, Phys. Rev. Lett. 92, 138301 (2004).

[9] M. Catanzaro, M. Boguna, and R. Pastor-Satorras, Phys. Rev. E. 71, 056104 (2005).

[10] S.H. Strogatz, Nature 410, 268 (2001).

[11] G. Korniss, M.A. Novotny, H. Guclu, Z. Toroczkai, and P.A. Rikvold, Science 299, 677 (2003).

[12] V.M. Eguíluz, D.R. Chialvo, G.A. Cecchi, M. Baliki, and A.V. Apkarian, Phys. Rev. Lett. 94, 018102 (2005).

[13] C.-W. Shin and S. Kim, cond-mat/0408700 (2004).

[14] J.J. Hopfield and C.D. Brody, Proc. Natl. Acad. Sci. U.S.A. 98,
1282 (2001).

[15] M. Barahona and L.M. Pecora, Phys. Rev. Lett. 89, 054101 (2002).

[16] T. Nishikawa, A.E. Motter, Y.-C. Lai, and F.C. Hoppensteadt, Phys. Rev. Lett. 91, 014101 (2003).

[17] H. Hong, B.J. Kim, M.Y. Choi, and H. Park, Phys. Rev. E 69 067105 (2004).

[18] Y. Kuramoto, in Proceedings of the International Symposium on Mathematical Problems in Theoretical Physics, edited by H. Araki (Springer-Verlag, New York, 1975); Chemical Oscillations, Waves, and Turbulence (Springer-Verlag, Berlin, 1984).

[19] S.H. Strogatz, Physica D 143, 1 (2000).

[20] H. Daido, J. Phys. A 20, L629 (1987).

[21] H. Hong, M.Y. Choi, and B.J. Kim, Phys. Rev. E. 65, 026139 (2002).

[22] P. Erdős and A. Rényi, Publ. Math. Inst. Hung. Acad. Sci. 5, 17 (1960); Bull. Inst. Int. Stat. 38, 343 (1961).

[23] K.-I. Goh, B. Kahng, and D. Kim, Phys. Rev. Lett. 87, 278701 (2001)

[24] D.-S. Lee,K.-I. Goh, B. Kahng, and D. Kim, Nucl. Phys. B 696, 351 (2004)

[25] For $p_{d}(k)=k^{-\lambda / \zeta(\lambda)}(k \geq 1)$, one finds that $f_{\lambda}(\infty)=$ $2^{-\lambda / 2-3 / 2}(\lambda / 2-3 / 2) !(-\lambda / 2) ! /[(\lambda / 2) ! \zeta(\lambda)]=\bar{f}_{\lambda+1}(\infty)$. 
[26] Y. Moreno and A.F. Pacheco, Europhys. Lett. 68, 603 (2004).

[27] R. Cohen, D. ben-Avraham, and S. Havlin, Phys. Rev. E 66, 036113 (2002); R. Cohen, S. Havlin, and D. ben-Avraham, in Handbook of Graphs and Networks, edited by S. Bornholdt and H.G. Shuster (Willey-VCH, New York, 2002), Chap. 4.
[28] C.P. Herrero, Phys. Rev. E 69, 067109 (2004).

[29] S.N. Dorogovtsev, A.V. Goltsev, and J.F.F. Mendes, Phys. Rev. E 66, 016104 (2002).

[30] T. Ichinomiya, Phys. Rev. E 70, 026116 (2004). 